\title{
Quantifying drivers of methane hydrobiogeochemistry in a tidal river floodplain
}

\author{
ZhangShuan HOU ${ }^{1}$, Nicholas D. WARD ${ }^{2}$, STEVEN B. \\ YABUSAKI $^{3 *}$, SCOTT R. WAICHLER ${ }^{4}$, MATTHEW \\ NORWOOD $^{5}$, ALLISON MYERS-PIGG ${ }^{6}$, JULIA INDIVERO ${ }^{7}$ \\ Pacific Northwest National Laboratory \\ ${ }^{1}$ zhangshuan.hou@pnnl.gov \\ 2nicholas.ward@pnnl.gov \\ ${ }^{3}$ yabusaki@pnnl.gov *presenting author \\ ${ }^{4}$ scott.waichler@pnnl.gov \\ 5matthew.norwood@pnnl.gov \\ 6allison.myers-pigg@pnnl.gov \\ 7julia.indivero@pnnl.gov
}

A large uncertainty in global greenhouse gas budgets is how increasing inundation and salinization of coastal freshwater ecosystems will affect terrestrial methane sources and sinks. Observations of methane behavior in a first-order tidal stream floodplain outline a potentially complex interplay between production, oxidation, and transport processes that are strongly affected by inundation events with seasonally variable salinity. A systematic and predictive understanding of methane behavior in coastal ecosystems undergoing increasing inundation and salinity exposure is needed to provide a basis for representing this phenomenon in earth system models.

An uncertainty quantification and machine learning (UQML) framework has been developed to identify and rank the most influential processes, properties, and conditions controlling methane behavior using a comprehensive multiyear dataset and mechanistic modeling. The dataset includes spatially sparse and temporally low to high frequency field observations of methane concentration and fluxes, salinity, temperature, depth to water table, water content, $\mathrm{pH}, \mathrm{CO}_{2}, \mathrm{O}_{2}$, DOC, total N, major ions, topography, hydraulic conductivity, and porosity, collected in and on the stream; in the groundwater, vadose zone, and on the ground surface; and on the ponded water during floodplain inundation.

We present a conceptual model of the spatiotemporal methane behavior in the tidal stream floodplain based on its relationships with site characteristics identified by the UQML framework. Methane transport, sources, and sinks dynamically respond to seasonal and interannual salinity exposure during quasi-monthly inundation events. This understanding will be used as the basis for three-dimensional methane reactive transport modeling of the tidal stream floodplain system. 